\title{
Information is meagre on aetiology of oral cancer in young people
}

\section{What are the risk factors associated with the development of oral cancer in young people?}

\begin{abstract}
Llewellyn CD, Johnson NW, Warnakulasuriya KASS. Risk factors for squamous cell carcinoma of the oral cavity in young people-a comprehensive literature review. Oral Oncol 2001; 37:401-418.
\end{abstract}

Data sources Searches were made of Medline, Cancerlit, the Institute for Scientific Information (ISI) databases of SCl-expanded and Social Sciences Citation Index (SciSearch), and of Embase (1980present) and the International Bibliography of the Social Sciences both via BIDS. Relevant journals and indices were searched by hand.

Study selection Only English-language articles published between 1957 and 2000 were included. Articles that did not include studies of squamous cell carcinoma or describing cancer of upper aerodigestive tract, oesophagus or larynx were excluded.

Data abstraction and synthesis A qualitative synthesis of the studies included was carried out.

Results A total of 46 articles exclusively considering young adults were identified, of which 28 examined potential risk factors. About 4$6 \%$ of oral cancers occur in people aged under 40 years. The usual male dominance does not appear to hold for younger patients. There are inconsistencies in the survival rates of younger patients compared with older patients but this may be due to small sample sizes and differences in treatment type and duration. Several studies suggest that many younger patients have never used tobacco or consumed alcohol or the duration of exposure is too short for malignant transformation too occur. There is insufficient research on association with occupation, immune defence, viral infections, diets low in fruit and vegetables or genetic factors.

Conclusions There is mixed evidence regarding the role of alcohol and tobacco as risk factors in young people. There is limited research on other risk factors.

\section{Commentary}

This systematic evaluation of the available literature provides a comprehensive survey of oral squamous cell carcinoma (OSCC) in young people, including risk factors for this disease. Using accepted search strategies, 46 articles were included of which 28 described potential risk factors. Of the latter, nine included control groups and only four included more than 50 subjects. These small numbers exemplify the difficulties that the authors had to face in identifying risk factors.

To begin with one of the conclusions: there is no general agreement for the age criteria of young people in this context. Despite this, it was shown that the incidence of OSCC in young people (up to 45 years of age) has doubled or even tripled in Europe, the US and, in particular, in the Indian subcotinent in recent years. There was less evidence for changes in gender distribution.

Although the increasing epidemiological burden of OSCC in younger individuals seems to be obvious, there is still a lack of identified risk factors. The review found controversial evidence for both a high and a low usage of tobacco and alcohol in young individuals with OSCC. There were some clues, however, that these tumouts could be associated with genetic factors, an inherent susceptibility to OSCC, for example, linked to polymorphisms or chromosome fragility. For any other known risk factors, such as betel quid chewing, occupational risks, viral infections and diet, no direct evidence was found. The authors therefore concluded that there is a definitive need for valid data to establish causative factors underlying oral cancer in the young. They announced a 3-year matched case-control study to be performed in the UK including 12 million people. In general, the results underline the necessity for further investigations using multicentre studies. These might be of particular importance in the high-risk areas of India and Asia.

\section{Practice points}

- The number of oral cancers in people of age $<45$ years has increased.

- Tobacco and alcohol use may be involved but clear risk factors are yet to be identified.

- More work is needed to establish risk factors for OSCC in younger age groups.

\section{Christian Scheifele, Peter A. Reichart}

Department of Oral Sugery and Dental Radiology, Medical Faculty Charité, Humboldt University, Berlin, Germany

Evidence-Based Dentistry (2003) 4, 7.

doi:10.1038/sj.ebd.6400153 